University of Nebraska - Lincoln

DigitalCommons@University of Nebraska - Lincoln

Faculty Publications, Department of Physics and Astronomy

Research Papers in Physics and Astronomy

$12-1-2004$

\title{
THE SPECTRA OF TYPE II CEPHEIDS. III. THE H $\alpha$ LINE AND HELIUM EMISSION IN LONG-PERIOD STARS
}

\author{
Edward G. Schmidt \\ University of Nebraska-Lincoln, eschmidt1@unl.edu \\ Dale Johnston \\ University of Nebraska-Lincoln, dalej96@gmail.com \\ Kevin M. Lee \\ University of Nebraska-Lincoln, klee6@unl.edu \\ Shawn Langan \\ University of Nebraska-Lincoln, nuaur@hotmail.com \\ Peter R. Newman \\ New Mexico State University, prn@apo.nmsu.edu
}

See next page for additional authors

Follow this and additional works at: https://digitalcommons.unl.edu/physicsfacpub

Part of the Physics Commons

Schmidt, Edward G.; Johnston, Dale; Lee, Kevin M.; Langan, Shawn; Newman, Peter R.; and Snedden, Stephanie A., "THE SPECTRA OF TYPE II CEPHEIDS. III. THE H $\alpha$ LINE AND HELIUM EMISSION IN LONGPERIOD STARS" (2004). Faculty Publications, Department of Physics and Astronomy. 25.

https://digitalcommons.unl.edu/physicsfacpub/25

This Article is brought to you for free and open access by the Research Papers in Physics and Astronomy at DigitalCommons@University of Nebraska - Lincoln. It has been accepted for inclusion in Faculty Publications, Department of Physics and Astronomy by an authorized administrator of DigitalCommons@University of Nebraska - Lincoln. 


\section{Authors}

Edward G. Schmidt, Dale Johnston, Kevin M. Lee, Shawn Langan, Peter R. Newman, and Stephanie A. Snedden 
The Astronomical Journal, 128:2988-2996, 2004 December

(C) 2004. The American Astronomical Society. All rights reserved. Printed in U.S.A.

\title{
THE SPECTRA OF TYPE II CEPHEIDS. III. THE H $\alpha$ LINE AND HELIUM EMISSION IN LONG-PERIOD STARS ${ }^{1}$
}

\author{
Edward G. Schmidt, ${ }^{2}$ Dale Johnston, ${ }^{2}$ Kevin M. Lee, and Shawn Langan ${ }^{2}$ \\ Department of Physics and Astronomy, University of Nebraska-Lincoln, Lincoln, NE 68588-0111; \\ eschmidt1@unl.edu, dalej96@bigred.unl.edu,klee6@unl.edu,nuaur@hotmail.com \\ AND \\ Peter R. Newman and Stephanie A. Snedden \\ Apache Point Observatory, P.O. Box 59, Sunspot, NM 88349; and Department of Astronomy, New Mexico State University, \\ P.O. Box 30001, Las Cruces, NM 88003-8001; prn@apo.nmsu.edu, snedden@apo.nmsu.edu \\ Received 2004 August 17; accepted 2004 August 26
}

\begin{abstract}
We have obtained $\mathrm{H} \alpha$ profiles from 175 spectra of 37 Cepheids with periods longer than 8 days. About twothirds of the stars are likely type II Cepheids. In contrast to the situation among short-period stars, the velocity of $\mathrm{H} \alpha$ relative to the metal-line spectrum is not a useful parameter for distinguishing type I and type II Cepheids. On the other hand, at periods longer than 11 days and shorter than 34 days strong emission is observed in many stars and appears to be a good discriminator of type. On this basis we identify one probable type I Cepheid at a large distance from the Galactic plane. We have found He $\lambda 5876$ emission in seven Cepheids among the long-period stars discussed here and in one star with a short period, bringing to nine the number of type II Cepheids known to exhibit He emission.
\end{abstract}

Key words: Cepheids - stars: Population II

\section{INTRODUCTION}

This is the third in a series of papers discussing the spectra of type II Cepheids as compared with those of type I or classical Cepheids. In Schmidt et al. (2003b, hereafter Paper I) we discussed the profiles of $\mathrm{H} \alpha$ for stars with periods shorter than 3 days. Previous work has shown that emission in $\mathrm{H} \alpha$ is uncommon among such stars and is weak when it does occur. In contrast, we found moderately strong emission in those type II Cepheids exhibiting a strong bump on the light curve during rising light. The emission always coincided with the bump. We also found large velocity differentials between $\mathrm{H} \alpha$ and the metal lines for many of the type II Cepheids. In Schmidt et al. (2003a, Paper II) stars in the intermediate range from 3 to 8 days were discussed. Although we had good phase coverage for a sizable sample of stars, no cases of emission at $\mathrm{H} \alpha$ were found. In addition, the differential velocities were generally small, leading us to question whether more than a few of the stars were bona fide type II Cepheids.

In the present paper we consider the behavior of $\mathrm{H} \alpha$ in Cepheids with periods longer than 8 days. More than half a century ago Joy (1949) found that hydrogen line emission is common among such stars in globular clusters. Others have since reported emission in various field type II Cepheids, including Wallerstein (1958), who reported emission in seven out of 11 stars, Harris \& Wallerstein (1984), who reported emission during rising light in 13 out of 20 stars, and Lèbre \& Gillet (1992), who followed the evolution of the emission profile of

\footnotetext{
${ }^{1}$ Based in part on observations obtained with the Apache Point Observatory $3.5 \mathrm{~m}$ telescope, which is owned and operated by the Astrophysical Research Consortium.

${ }^{2}$ Visiting Astronomer, Kitt Peak National Observatory, National Optical Astronomy Observatory, which is operated by the Association of Universities for Research in Astronomy, Inc., under cooperative agreement with the National Science Foundation (NSF).
}

$\mathrm{H} \alpha$ through the cycle in the prototype long-period type II Cepheid W Vir. Various authors have also measured the differential velocity of $\mathrm{H} \alpha$ relative to the photospheric metal lines, $\Delta \mathrm{Vel}=V_{\mathrm{H} \alpha}-V_{\mathrm{ph}}$, in long-period Cepheids (summarized by Vinkó et al. 1998). It has been found that as the periods increase beyond about 8 days increasingly large values of $\Delta$ Vel are observed for both classical and type II Cepheids although the number of the latter was very limited. We will extend this previous work by presenting such information for a much larger sample of stars.

Wallerstein (1959) reported emission in the neutral helium lines at 5876 and $6678 \AA$ in the spectrum of W Vir during rising light. Subsequently, higher quality spectra were obtained by Raga et al. (1989) and Lèbre \& Gillet (1992) to study the behavior of both hydrogen and helium emission in W Vir. In this paper we take advantage of our large sample of stars in discussing the prevalence of $\mathrm{He}$ emission among Cepheids.

\section{THE OBSERVATIONS}

\subsection{The Sample of Stars}

The stars discussed here are listed in Table 1, in which column (1) gives the names of the stars and column (2) gives the approximate periods. Column (3) contains the classifications from the General Catalogue of Variable Stars (Kholopov 1985, 1987; hereafter GCVS). A "II" in column (4) indicates that the star appears in Table I of Harris's (1985) catalog of type II Cepheids. He selected these objects on the basis of distance from the Galactic plane. Hence, we will refer to the stars listed by Harris as high- $z$ stars and the remainder as low- $z$ stars, as we did previously in Schmidt et al. (2004). Column (5) of Table 1 lists the metallicities taken from Harris (1981a, 1981b), Harris \& Wallerstein (1984), or Meakes et al. (1991).

Our list includes about two-thirds of the stars classified as long-period type II Cepheids in the GCVS that are bright 
TABLE 1

The Program Stars

\begin{tabular}{|c|c|c|c|c|}
\hline $\begin{array}{l}\text { Star } \\
(1)\end{array}$ & $\begin{array}{c}\text { Period } \\
\text { (days) } \\
(2)\end{array}$ & $\begin{array}{c}\text { GCVS Class } \\
\text { (3) }\end{array}$ & $\begin{array}{c}\text { Harris Class } \\
\text { (4) }\end{array}$ & $\begin{array}{c}{[\mathrm{Fe} / \mathrm{H}]} \\
(5)\end{array}$ \\
\hline PZ Aql............ & 8.8 & CWA & $\ldots$ & \\
\hline IX Cas ............. & 9.1 & CWA & II & $-0.4,0.3$ \\
\hline CN Cep ........... & 9.5 & DCEP & $\ldots$ & \\
\hline AN Aur ........... & 10.3 & DCEP & $\ldots$ & -0.2 \\
\hline AL Vir ............. & 10.3 & CWA & II & $-0.7,-0.5$ \\
\hline AP Her ............ & 10.4 & CWA & II & $-0.8,-1.8$ \\
\hline BH Oph.......... & 11.1 & CWA & II & \\
\hline QQ Per ........... & 11.2 & CEP & II & \\
\hline AD Cam.......... & 11.3 & DCEP & $\ldots$ & -1.0 \\
\hline EZ Cyg............ & 11.7 & DCEP & $\ldots$ & \\
\hline V343 Cyg....... & 11.9 & CWA & II & -0.2 \\
\hline V775 Oph ...... & 12.2 & CWA & II & \\
\hline AS Vul ........... & 12.2 & DCEP & $\ldots$ & \\
\hline AL Lyr ........... & 13.0 & CWA & II & \\
\hline V916 Aq1 ....... & 13.4 & DCEP & $\ldots$ & \\
\hline V801 Aql ....... & 14.2 & CWA & II & \\
\hline V833 Oph ...... & 14.7 & CWA & II & \\
\hline CS Cas ............ & 14.7 & CWA & II & -0.4 \\
\hline EU Cyg .......... & 15.0 & CEP & II & \\
\hline CH Cas............ & 15.1 & DCEP & $\ldots$ & -0.5 \\
\hline V845 Her ........ & 15.5 & CWA & $\ldots$ & \\
\hline SZ Mon ........... & 16.3 & RVA & $\ldots$ & \\
\hline V478 Oph ...... & 16.3 & CWA & II & -0.7 \\
\hline CP Сep............ & 17.9 & DCEP & $\ldots$ & \\
\hline DR Cep .......... & 19.1 & DCEP & II & -0.1 \\
\hline KX Cyg.......... & 20.1 & DCEP & $\ldots$ & \\
\hline MZ Cyg.......... & 21.4 & CWA: & II & 0.1 \\
\hline PP Aql ............ & 24.0 & DCEP & II & 0.0 \\
\hline CC Lyr ............ & 24.2 & CWA & II & $-2.2,-2.3$ \\
\hline OT Per............. & 26.1 & DCEP & $\ldots$ & \\
\hline TW Cap.......... & 28.6 & CWA & II & -1.2 \\
\hline NN Vul........... & 30.8 & CWA & $\ldots$ & \\
\hline V609 Cyg....... & 31.1 & DCEP & $\ldots$ & \\
\hline IU Cyg ............ & 31.4 & CW: & II & \\
\hline V1281 Aql..... & 32.0 & CWA: & II & \\
\hline EV Aql ........... & 38.8 & CEP & II & -0.3 \\
\hline ET Vul............. & 54.5 & CWA & II & -0.4 \\
\hline
\end{tabular}

enough and far enough north for feasible spectral and photometric observations. To these we have added several that may be of this type and a dozen classical Cepheids for comparison purposes.

\subsection{The Data}

The spectra were obtained at Kitt Peak National Observatory during 2001 July and 2002 September/October and at Apache Point Observatory during 2002 June. They are of intermediate resolution with FWHM $=2.0 \AA$ for the KPNO spectra and $\mathrm{FWHM}=2.6 \AA$ for the APO spectra. The data presented in Papers I and II were taken during the same observing runs as those presented here. Paper I should be consulted for a detailed description of the data and the procedures used in the observations and reductions. ${ }^{3}$

A $\log$ of the spectroscopic observations is given in Table 2, where the stars are identified in column (1) and the Heliocentric Julian Date of mid-exposure is given in column (2). The

\footnotetext{
${ }^{3}$ All of the reduced spectra are available at http://astro.unl.edu/observatory/ data/cepheids.html.
}

spectra from Apache Point Observatory can be distinguished by having Julian Dates of 2,452,442 and 2,452,443, while the remaining dates indicate Kitt Peak spectra. The phases of the spectra relative to maximum light are listed in column (3). For all except three of the stars, we used the elements given by Schmidt et al. (2004) to calculate the phases. These are based on photometry that overlaps our spectra in time, so the phases will be quite reliable. For the three stars in the present sample that were not included in Schmidt et al., we have estimated the phases as indicated in footnotes to Table 2.

As in Papers I and II, we tried to concentrate our observations during rising light and shortly afterward. In spite of poor ephemerides for some stars at the time of the observations, about $70 \%$ of the spectra fall between minimum light (as given in Table 3 of Schmidt et al. 2004) and 0.05 cycles after maximum.

We again used the rms scatter in the region from 6720 to $6820 \AA$ to derive a lower estimate of the signal-to-noise ratio $(\mathrm{S} / \mathrm{N})$ for the spectra. We achieved a $\mathrm{S} / \mathrm{N}$ greater than 100 per resolution element for $40 \%$ of the spectra and greater than 75 for three-quarters of them. In a few cases in which the $\mathrm{S} / \mathrm{N}$ was below 50, we have used the spectra but marked them with a footnote in Table 2.

\subsection{The Profile of $\mathrm{H} \alpha$}

In Figure 1 we show examples of $\mathrm{H} \alpha$ profiles that are dominated by absorption. While most show no apparent emission, the wide range of strength and depth suggests filling by emission in some cases. We also note that the wings vary considerably even among those stars with strong $\mathrm{H} \alpha$. In the spectrum of SZ Mon there appears to be emission in both wings. This is unique among our spectra, but it is similar to some of the spectra of W Vir shown by Lèbre \& Gillet (1992). The spectrum of AL Lyr might be construed in terms of an inverse P Cyg profile with emission on the shortward wing. A half-dozen similar profiles are to be found among our spectra, but a careful examination of them suggests that an absorption line, probably $\mathrm{Ti}$ II 26559.576 , may depress the shortward wing, giving the erroneous impression of emission. In fact, that line is evident in many of our spectra that lack emission (including several in Fig. 1). Taking that possibility into account, none of the possible inverse P Cyg profiles were judged to be convincing. Hence, we have not identified any stars as having such a profile, in spite of the obvious inverse P Cyg profile visible in some of the spectra of W Vir (Lèbre \& Gillet 1992). In column (4) of Table 2 we identify the spectra with profiles like those labeled in Figure 1 with the letter "A."

Many of our spectra exhibit strong $\mathrm{H} \alpha$ emission features, and we provide examples in Figure 2. In the top spectrum, CC Lyr at phase 0.73 , emission completely dominates the profile. Such cases are denoted by an "E" in column (4). In the spectra of CS Cas at 0.05 and CC Lyr at 0.01 absorption wings are visible on either side of the emission feature. Profiles of this type are denoted by a "wE" in column (4). V478 Oph at 0.92 , V775 Oph at 0.56, and SZ Mon at 0.53 exhibit strong emission which is split into two components (indicated by "dE"). Finally, the spectrum of PP Aql at 0.15 in Figure 2 lacks $\mathrm{H} \alpha$ and is likely to be due to a cancellation of emission and absorption.

Columns (5) and (6) of Table 2 present the differential velocity of $\mathrm{H} \alpha$ relative to the metal lines and its standard error. These quantities were determined in the same way as described in Paper I. For CC Lyr, which is very metal-poor, the metal lines were too weak to measure, so no differential velocities are listed. The line depth, or in the case of an emission feature, the 
TABLE 2

JoURNAL OF OBSERVATIONS

\begin{tabular}{|c|c|c|c|c|c|c|c|}
\hline $\begin{array}{l}\text { Star } \\
(1)\end{array}$ & $\begin{array}{c}\text { Mid-Exposure HJD }-2,452,000 \\
\text { (2) }\end{array}$ & $\begin{array}{c}\phi \\
(3)\end{array}$ & $\begin{array}{l}\text { Form of Profile } \\
\text { (4) }\end{array}$ & $\begin{array}{c}\Delta \mathrm{Vel} \\
\left(\mathrm{km} \mathrm{s}^{-1}\right) \\
(5)\end{array}$ & $\begin{array}{c}\text { Std. Err. } \\
\left(\mathrm{km} \mathrm{s}^{-1}\right) \\
(6)\end{array}$ & $\begin{array}{l}\text { Depth of } \mathrm{H} \alpha \\
\text { (7) }\end{array}$ & $\begin{array}{c}W_{\lambda} \text { of } \mathrm{H} \alpha \\
(\AA) \\
(8)\end{array}$ \\
\hline \multirow[t]{3}{*}{ PZ Aql.............. } & 106.74 & 0.50 & A & -31 & 3 & 0.55 & 3.2 \\
\hline & 547.67 & 0.86 & $\mathrm{~A}$ & 10 & 3 & 0.57 & 4.0 \\
\hline & 554.60 & 0.66 & A & 11 & 3 & 0.49 & 2.4 \\
\hline \multirow[t]{9}{*}{ IX Cas................. } & 104.81 & 0.72 & A & -6 & 3 & 0.46 & 3.3 \\
\hline & 105.79 & 0.83 & $\mathrm{~A}$ & -12 & 3 & 0.48 & 3.7 \\
\hline & 106.78 & 0.94 & A & -15 & 3 & 0.51 & 3.8 \\
\hline & 546.95 & 0.05 & A & -8 & 4 & 0.50 & 3.2 \\
\hline & 551.86 & 0.59 & $\mathrm{~A}$ & -13 & 3 & 0.46 & 2.3 \\
\hline & 553.85 & 0.81 & A & -7 & 2 & 0.52 & 3.3 \\
\hline & 554.87 & 0.91 & A & -9 & 2 & 0.54 & 3.5 \\
\hline & 555.83 & 0.02 & A & -19 & 4 & 0.51 & 3.3 \\
\hline & 555.89 & 0.03 & A & -8 & 3 & 0.52 & 3.5 \\
\hline \multirow[t]{5}{*}{ CN Cep ................ } & 101.96 & 0.87 & A & -4 & 3 & 0.62 & 3.4 \\
\hline & 104.83 & 0.17 & A & -18 & 3 & 0.56 & 3.6 \\
\hline & 547.74 & 0.77 & A & -1 & 2 & 0.54 & 2.4 \\
\hline & 548.81 & 0.89 & A & 2 & 2 & 0.63 & 3.4 \\
\hline & 551.97 & 0.22 & A & -5 & 3 & 0.58 & 3.4 \\
\hline \multirow[t]{5}{*}{ AN Aur ............. } & 546.99 & 0.21 & A & -4 & 3 & 0.58 & 2.8 \\
\hline & 550.79 & 0.58 & A & 20 & 3 & 0.50 & 2.1 \\
\hline & 551.78 & 0.67 & $\mathrm{~A}$ & 43 & 3 & 0.59 & 3.1 \\
\hline & 553.83 & 0.87 & A & -26 & 3 & 0.55 & 4.1 \\
\hline & 555.86 & 0.07 & A & -7 & 3 & 0.57 & 3.2 \\
\hline AL Vir ................ & 442.66 & 0.77 & A & 24 & 4 & 0.39 & 1.9 \\
\hline \multirow[t]{5}{*}{ AP Her .............. } & 105.91 & 0.87 & A & 20 & 3 & 0.28 & 1.2 \\
\hline & 106.67 & 0.94 & A & 28 & 4 & 0.39 & 2.2 \\
\hline & $550.73^{\mathrm{a}}$ & 0.64 & $\mathrm{~A}$ & 20 & 14 & 0.50 & 1.8 \\
\hline & 553.72 & 0.92 & A & 35 & 3 & 0.38 & 2.0 \\
\hline & 554.73 & 0.02 & A & 9 & 3 & 0.26 & 2.6 \\
\hline \multirow[t]{4}{*}{ BH Oph........... } & 105.91 & 0.67 & A & 3 & 3 & 0.38 & 1.2 \\
\hline & 106.80 & 0.75 & A & 2 & 4 & 0.34 & 1.1 \\
\hline & 548.70 & 0.71 & A & 1 & 4 & 0.38 & 1.0 \\
\hline & 550.69 & 0.89 & $\mathrm{~A}$ & 33 & 5 & 0.36 & 1.0 \\
\hline \multirow[t]{4}{*}{ QQ Per ............. } & 101.93 & 0.00 & $\mathrm{wE}$ & -21 & 4 & -0.26 & -0.07 \\
\hline & 546.74 & 0.76 & $\mathrm{~A}$ & 48 & 5 & 0.52 & 3.7 \\
\hline & 547.75 & 0.85 & $\mathrm{E}$ & -31 & 4 & -0.77 & -2.7 \\
\hline & 555.98 & 0.58 & $w d E$ & $-64,11,64$ & 4 & $-0.09,-0.05,0.02$ & 0.2 \\
\hline \multirow[t]{6}{*}{ AD Cam............ } & 105.97 & 0.66 & A & -41 & 4 & 0.47 & 2.2 \\
\hline & 106.91 & 0.74 & A & -27 & 4 & 0.42 & 2.1 \\
\hline & 546.78 & 0.80 & A & 16 & 3 & 0.38 & 1.8 \\
\hline & 547.76 & 0.89 & A & 23 & 2 & 0.59 & 3.0 \\
\hline & 548.85 & 0.98 & A & 12 & 3 & 0.62 & 4.2 \\
\hline & 555.99 & 0.62 & A & -28 & 3 & 0.43 & 1.7 \\
\hline \multirow{5}{*}{ EZ Cyg............... } & 102.81 & 0.68 & A & 15 & 4 & 0.43 & 1.7 \\
\hline & 104.83 & 0.85 & A & 10 & 3 & 0.64 & 2.7 \\
\hline & 105.82 & 0.93 & A & 9 & 3 & 0.64 & 2.6 \\
\hline & 106.86 & 0.02 & A & 16 & 3 & 0.60 & 3.2 \\
\hline & 551.79 & 0.18 & A & 16 & 3 & 0.58 & 2.6 \\
\hline \multirow{6}{*}{ V343 Cyg ${ }^{\mathrm{b}} \ldots .}$. & 104.92 & 0.66 : & A & -35 & 4 & 0.43 & 1.4 \\
\hline & 105.71 & 0.73: & A & -35 & 3 & 0.40 & 1.3 \\
\hline & 106.68 & $0.81:$ & $\mathrm{A}$ & 5 & 3 & 0.41 & 1.5 \\
\hline & 546.83 & 0.71: & A & -19 & 3 & 0.44 & 1.4 \\
\hline & 550.78 & $0.04:$ & A & 32 & 2 & 0.60 & 2.4 \\
\hline & 551.80 & $0.13:$ & A & 30 & 2 & 0.60 & 2.9 \\
\hline \multirow[t]{5}{*}{ V775 Oph ....... } & 101.85 & 0.96 & A & -5 & 3 & 0.36 & 1.1 \\
\hline & 106.66 & 0.36 & A & -23 & 3 & 0.25 & 0.6 \\
\hline & 547.66 & 0.56 & $\mathrm{dE}$ & $-67,-28,34$ & 6 & $-0.17,-0.10,-0.32$ & -1.1 \\
\hline & 550.63 & 0.81 & A & -4 & 4 & 0.27 & 0.6 \\
\hline & 551.70 & 0.90 & A & -5 & 4 & 0.31 & 1.2 \\
\hline \multirow[t]{5}{*}{ AS Vul .............. } & 101.94 & 0.96 & A & 9 & 2 & 0.57 & 2.1 \\
\hline & 102.85 & 0.03 & A & 23 & 3 & 0.56 & 2.4 \\
\hline & 551.59 & 0.74 & $\mathrm{~A}$ & -22 & 4 & 0.46 & 1.6 \\
\hline & 553.72 & 0.91 & $\mathrm{~A}$ & 4 & 3 & 0.66 & 2.7 \\
\hline & 554.69 & 0.99 & A & 12 & 3 & 0.64 & 3.0 \\
\hline
\end{tabular}


TABLE 2-Continued

\begin{tabular}{|c|c|c|c|c|c|c|c|}
\hline $\begin{array}{l}\text { Star } \\
(1)\end{array}$ & $\begin{array}{c}\text { Mid-Exposure HJD }-2,452,000 \\
\text { (2) }\end{array}$ & $\begin{array}{c}\phi \\
(3)\end{array}$ & $\begin{array}{l}\text { Form of Profile } \\
\text { (4) }\end{array}$ & $\begin{array}{c}\Delta \mathrm{Vel} \\
\left(\mathrm{km} \mathrm{s}^{-1}\right) \\
(5)\end{array}$ & $\begin{array}{c}\text { Std. Err. } \\
\left(\mathrm{km} \mathrm{s}^{-1}\right) \\
(6)\end{array}$ & $\begin{array}{l}\text { Depth of } \mathrm{H} \alpha \\
\text { (7) }\end{array}$ & $\begin{array}{c}W_{\lambda} \text { of } \mathrm{H} \alpha \\
(\AA) \\
(8)\end{array}$ \\
\hline \multirow{8}{*}{ AL Lyr ............. } & 101.91 & 0.53 & $\mathrm{dE}$ & $-89,-19,94$ & 3 & $-0.08,0.03,-0.11$ & -0.5 \\
\hline & 102.80 & 0.60 & $\mathrm{dE}$ & $-64,-2,24$ & 4 & $-0.94,-0.85,-0.89$ & -5.4 \\
\hline & 103.84 & 0.68 & $\mathrm{E}$ & -46 & 4 & -0.98 & -5.3 \\
\hline & 104.90 & 0.76 & $\mathrm{E}$ & -43 & 4 & -0.74 & -2.9 \\
\hline & 105.85 & 0.84 & $w E$ & -56 & 4 & -0.26 & -0.6 \\
\hline & 106.91 & 0.92 & $\mathrm{~A}$ & 35 & 3 & 0.20 & 0.9 \\
\hline & 546.67 & 0.80 & $\mathrm{E}$ & -40 & 4 & -0.15 & -0.3 \\
\hline & 551.77 & 0.20 & A & -1 & 4 & 0.50 & 1.6 \\
\hline \multirow[t]{5}{*}{ V916 Aq1 ........ } & $104.87^{\mathrm{a}}$ & 0.65 & $\mathrm{~A}$ & -9 & 3 & 0.36 & 1.4 \\
\hline & 105.76 & 0.71 & A & -11 & 4 & 0.37 & 1.4 \\
\hline & 106.79 & 0.79 & A & -1 & 3 & 0.38 & 1.7 \\
\hline & 550.75 & 0.82 & A & 3 & 4 & 0.47 & 2.1 \\
\hline & 554.74 & 0.11 & A & 34 & 2 & 0.63 & 3.8 \\
\hline \multirow[t]{4}{*}{ V801 Aq1 ........ } & 105.84 & 0.96 & A & 11 & 6 & 0.28 & 0.8 \\
\hline & 106.89 & 0.03 & A & -6 & 2 & 0.30 & 1.3 \\
\hline & 553.61 & 0.58 & $\mathrm{dE}$ & $-45,-30,21$ & 3 & $-0.54,-0.52,-0.62$ & -2.5 \\
\hline & 554.61 & 0.65 & $\mathrm{dE}$ & $-33,28,5$ & 3 & $-0.55,-0.54,-0.56$ & -2.3 \\
\hline \multirow[t]{3}{*}{ V833 $\mathrm{Oph}^{\mathrm{c}} \ldots .}$. & $442.78^{\mathrm{a}}$ & $0.05:$ & A & -30 & 3 & 0.45 & 2.6 \\
\hline & 443.78 & 0.12 : & A & 3 & 3 & 0.50 & 2.4 \\
\hline & $554.65^{\mathrm{a}}$ & 0.66 : & A & 20 & 4 & 0.41 & 1.3 \\
\hline \multirow{6}{*}{ CS Cas ................ } & 104.80 & 0.70 & $\mathrm{dE}$ & $-80,-57,-32$ & 6 & $-1.49,-1.46,-1.48$ & -10.7 \\
\hline & 105.78 & 0.77 & $\mathrm{E}$ & -56 & 4 & -1.46 & -9.9 \\
\hline & 106.78 & 0.84 & $\mathrm{E}$ & -96 & 6 & -0.89 & -4.6 \\
\hline & 546.79 & 0.70 & $\mathrm{E}$ & -73 & 5 & -2.22 & -11.9 \\
\hline & 547.78 & 0.77 & $\mathrm{E}$ & -73 & 7 & -2.11 & -10.1 \\
\hline & 551.99 & 0.05 & $w E$ & -22 & 3 & -0.23 & 1.0 \\
\hline EU Cyg ........... & 443.74 & 0.18 & A & 11 & 2 & 0.56 & 2.9 \\
\hline \multirow[t]{8}{*}{$\mathrm{CH}$ Cas............... } & 101.95 & 0.70 & A & -6 & 3 & 0.59 & 2.4 \\
\hline & 102.86 & 0.76 & A & -4 & 3 & 0.59 & 3.0 \\
\hline & 104.95 & 0.90 & A & -2 & 3 & 0.66 & 3.7 \\
\hline & 105.88 & 0.96 & A & -13 & 3 & 0.60 & 4.0 \\
\hline & 546.94 & 0.19 & $\mathrm{~A}$ & -13 & 3 & 0.59 & 3.0 \\
\hline & 551.98 & 0.52 & A & -12 & 3 & 0.47 & 2.0 \\
\hline & 553.86 & 0.65 & A & -3 & 3 & 0.47 & 1.8 \\
\hline & 555.84 & 0.78 & A & 10 & 3 & 0.56 & 2.6 \\
\hline V845 Her ........ & 106.87 & 0.30 & A & -7 & 5 & 0.26 & 0.7 \\
\hline \multirow[t]{2}{*}{ SZ Mon............ } & 551.01 & 0.23 & A & 17 & 2 & 0.25 & 0.3 \\
\hline & 555.92 & 0.53 & $\mathrm{dE}$ & $-89,4,93$ & 5 & $-0.39,-0.05,-0.18$ & -2.4 \\
\hline \multirow[t]{5}{*}{ V478 Oph ....... } & 101.86 & 0.68 & $\mathrm{E}$ & -23 & 4 & -1.44 & -6.5 \\
\hline & 102.78 & 0.73 & $\mathrm{E}$ & -27 & 4 & -1.04 & -4.5 \\
\hline & 104.70 & 0.85 & $\mathrm{E}$ & -33 & 3 & -.55 & -2.3 \\
\hline & 105.80 & 0.92 & $\mathrm{dE}$ & $-39,27,42$ & 4 & $-0.32,-0.19,-0.20$ & -1.3 \\
\hline & 106.85 & 0.98 & A & -14 & 7 & 0.56 & 4.3 \\
\hline \multirow{9}{*}{ СР Сеp............. } & 104.71 & 0.65 & A & -113 & 4 & 0.58 & 3.0 \\
\hline & 105.77 & 0.71 & A & -95 & 4 & 0.55 & 2.8 \\
\hline & 106.79 & 0.76 & A & -91 & 3 & 0.50 & 2.9 \\
\hline & 546.89 & 0.40 & A & -32 & 4 & 0.63 & 2.3 \\
\hline & 550.77 & 0.62 & A & -31 & 5 & 0.53 & 2.2 \\
\hline & 551.77 & 0.67 & A & -31 & 4 & 0.53 & 2.2 \\
\hline & 553.85 & 0.79 & A & -8 & 3 & 0.54 & 2.2 \\
\hline & 554.72 & 0.84 & A & -6 & 3 & 0.59 & 2.7 \\
\hline & 555.84 & 0.90 & A & -2 & 2 & 0.61 & 3.0 \\
\hline \multirow[t]{5}{*}{ DR Cep ............ } & 104.96 & 0.87 & A & 29 & 4 & 0.11 & 0.4 \\
\hline & 105.92 & 0.92 & A & 12 & 3 & 0.43 & 1.8 \\
\hline & 546.87 & 0.04 & A & 47 & 3 & 0.61 & 3.4 \\
\hline & 551.86 & 0.30 & $\mathrm{~A}$ & 6 & 3 & 0.54 & 2.3 \\
\hline & 555.88 & 0.51 & A & -20 & 3 & 0.50 & 1.8 \\
\hline \multirow[t]{5}{*}{ KX Cyg............... } & 105.72 & 0.76 & A & -29 & 6 & 0.41 & 1.5 \\
\hline & 106.74 & 0.82 & A & -17 & 5 & 0.40 & 1.6 \\
\hline & 546.71 & 0.76 & A & -28 & 4 & 0.47 & 2.6 \\
\hline & 547.70 & 0.81 & A & -24 & 4 & 0.47 & 1.8 \\
\hline & 548.77 & 0.86 & A & -2 & 4 & 0.46 & 1.7 \\
\hline
\end{tabular}


TABLE 2-Continued

\begin{tabular}{|c|c|c|c|c|c|c|c|}
\hline $\begin{array}{l}\text { Star } \\
(1)\end{array}$ & $\begin{array}{c}\text { Mid-Exposure HJD }-2,452,000 \\
\text { (2) }\end{array}$ & $\begin{array}{l}\phi \\
(3)\end{array}$ & $\begin{array}{l}\text { Form of Profile } \\
\text { (4) }\end{array}$ & $\begin{array}{c}\Delta \mathrm{Vel} \\
\left(\mathrm{km} \mathrm{s}^{-1}\right) \\
(5)\end{array}$ & $\begin{array}{c}\text { Std. Err. } \\
\left(\mathrm{km} \mathrm{s}^{-1}\right) \\
(6)\end{array}$ & $\begin{array}{l}\text { Depth of } \mathrm{H} \alpha \\
\text { (7) }\end{array}$ & $\begin{array}{c}W_{\lambda} \text { of } \mathrm{H} \alpha \\
(\AA) \\
(8)\end{array}$ \\
\hline \multirow[t]{6}{*}{ MZ Cyg............... } & 546.86 & 0.44 & A & -12 & 5 & 0.41 & 1.8 \\
\hline & 550.79 & 0.63 & $\mathrm{~A}$ & -18 & 4 & 0.36 & 1.4 \\
\hline & 551.76 & 0.67 & A & -16 & 4 & 0.28 & 1.1 \\
\hline & 553.85 & 0.77 & $\mathrm{E}$ & -16 & 4 & -0.26 & -1.1 \\
\hline & 554.71 & 0.81 & $\mathrm{E}$ & $-35^{\mathrm{d}}$ & 5 & -0.32 & -1.6 \\
\hline & 555.83 & 0.86 & $\mathrm{dE}$ & $-60,6,43$ & 4 & $-0.35,-0.24,-0.28$ & -2.0 \\
\hline \multirow[t]{6}{*}{ 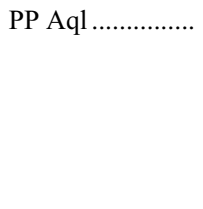 } & 101.95 & 0.02 & $\mathrm{E}$ & -21 & 3 & -0.51 & -1.3 \\
\hline & 102.85 & 0.06 & $\mathrm{E}$ & -18 & 4 & -0.33 & -0.9 \\
\hline & 104.95 & 0.15 & e & $\ldots$ & $\ldots$ & 0.00 & 0.0 \\
\hline & 105.90 & 0.19 & e & $\ldots$ & $\ldots$ & 0.00 & 0.0 \\
\hline & 550.76 & 0.68 & E & -7 & 4 & -0.64 & -3.1 \\
\hline & 554.72 & 0.85 & $\mathrm{E}$ & -18 & 6 & -0.57 & -2.1 \\
\hline \multirow[t]{7}{*}{ CC $\operatorname{Lyr}^{\mathrm{f}} \ldots \ldots \ldots \ldots \ldots$} & 101.90 & 0.61 & $\mathrm{E}$ & $\ldots$ & $\ldots$ & -0.34 & -1.1 \\
\hline & 102.79 & 0.65 & $\mathrm{E}$ & $\ldots$ & $\ldots$ & -0.47 & -1.3 \\
\hline & 103.83 & 0.69 & $\mathrm{E}$ & $\ldots$ & $\ldots$ & -0.47 & -1.4 \\
\hline & 104.89 & 0.73 & $\mathrm{E}$ & $\ldots$ & $\ldots$ & -0.57 & -1.7 \\
\hline & 105.84 & 0.77 & $\mathrm{E}$ & $\ldots$ & $\ldots$ & -0.57 & -1.6 \\
\hline & 106.89 & 0.82 & $\mathrm{E}$ & $\ldots$ & $\ldots$ & -0.60 & -1.7 \\
\hline & 547.76 & 0.01 & $w E$ & $\ldots$ & $\ldots$ & -0.31 & 0.4 \\
\hline \multirow[t]{3}{*}{ OT Per....................... } & 546.97 & 0.64 & A & -29 & 3 & 0.47 & 1.2 \\
\hline & 553.81 & 0.90 & A & -21 & 4 & 0.39 & 1.2 \\
\hline & 555.81 & 0.98 & A & 35 & 2 & 0.39 & 1.8 \\
\hline \multirow[t]{5}{*}{ TW Cap................ } & 546.69 & 0.75 & $\mathrm{dE}$ & $-91,-16,93$ & 5 & $-0.06,0.00,0.07$ & -0.1 \\
\hline & 547.68 & 0.79 & $\mathrm{dE}$ & $-82,-21,18$ & 4 & $-0.23,-0.09,-0.12$ & -0.7 \\
\hline & $550.72^{\mathrm{a}}$ & 0.89 & $\mathrm{E}$ & -27 & 3 & -0.72 & -2.9 \\
\hline & 553.66 & 0.00 & $w E$ & -58 & 5 & -0.43 & -1.4 \\
\hline & 554.67 & 0.03 & $w E$ & -50 & 5 & -0.27 & -0.1 \\
\hline NN Vul.................... & 102.83 & 0.98 & $\mathrm{E}$ & 47 & 5 & -0.12 & -0.2 \\
\hline \multirow[t]{6}{*}{ V609 Cyg................ } & 546.72 & 0.93 & A & 6 & 3 & 0.57 & 2.3 \\
\hline & 547.73 & 0.96 & A & 11 & 3 & 0.58 & 3.1 \\
\hline & 548.77 & 0.00 & $\mathrm{~A}$ & 19 & 3 & 0.63 & 3.7 \\
\hline & 550.87 & 0.06 & $\mathrm{~A}$ & 16 & 4 & 0.63 & 4.0 \\
\hline & 551.88 & 0.10 & A & 20 & 2 & 0.62 & 4.0 \\
\hline & 555.89 & 0.22 & A & -3 & 4 & 0.58 & 3.2 \\
\hline \multirow[t]{4}{*}{ IU Cyg ................... } & 546.70 & 0.95 & $\mathrm{E}$ & -54 & 5 & -0.84 & -3.7 \\
\hline & 547.68 & 0.98 & $\mathrm{E}$ & -60 & 3 & -0.69 & -2.7 \\
\hline & 548.76 & 0.02 & $\mathrm{E}$ & -36 & 4 & -0.60 & -2.2 \\
\hline & 550.83 & 0.08 & $\mathrm{E}$ & -36 & 4 & -0.55 & -1.8 \\
\hline \multirow[t]{2}{*}{ V1281 Aql ${ }^{\mathrm{g}} \ldots \ldots$} & 105.92 & 0.76 : & A & -3 & 5 & 0.19 & 0.6 \\
\hline & 106.91 & 0.79 : & A & -2 & 5 & 0.22 & 0.5 \\
\hline \multirow[t]{4}{*}{ 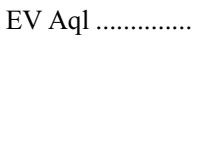 } & 104.98 & 0.79 & $\mathrm{~A}$ & -10 & 4 & 0.49 & 2.1 \\
\hline & 442.74 & 0.50 & A & -5 & 2 & 0.37 & 2.4 \\
\hline & 443.70 & 0.52 & $\mathrm{~A}$ & -7 & 3 & 0.41 & 2.1 \\
\hline & 554.73 & 0.39 & $\mathrm{~A}$ & -21 & 3 & 0.50 & 2.6 \\
\hline \multirow[t]{6}{*}{ ET Vul...................... } & 101.92 & 0.01 & A & 10 & 3 & 0.54 & 2.8 \\
\hline & 102.80 & 0.02 & A & 7 & 3 & 0.53 & 2.7 \\
\hline & 103.85 & 0.04 & A & 10 & 3 & 0.51 & 2.8 \\
\hline & 104.90 & 0.06 & A & 15 & 2 & 0.55 & 3.0 \\
\hline & 105.86 & 0.08 & A & 10 & 3 & 0.54 & 3.1 \\
\hline & 106.93 & 0.10 & A & 19 & 4 & 0.54 & 3.0 \\
\hline
\end{tabular}

a The lower limit to the $\mathrm{S} / \mathrm{N}$ is less than 50 .

b The most recent photometry of V343 Cyg was published by Schmidt \& Seth (1996), and we have used their elements for this star. Since fewer than 200 cycles elapsed since the end of their photometry and the beginning of our spectra, the phases should still be reasonably reliable.

${ }^{c}$ For V833 Oph the only relatively recent photometry is that of Berdnikov (1993). We derived an epoch of maximum of HJD $=2,447,738.7$ and a period of 14.698 days from that data. Since the photometry spanned the interval of about 200 cycles and another 300 cycles elapsed from the end of the photometry until the start of our spectral observations, the phases should be reasonably accurate.

${ }^{\mathrm{d}}$ In the spectrum of MZ Cyg at $\phi=0.81$ there is a shoulder at a differential velocity of about $30 \mathrm{~km} \mathrm{~s}^{-1}$ suggesting incipient doubling.

e No $\mathrm{H} \alpha$ emission or absorption is visible.

${ }^{\mathrm{f}}$ The metal lines in CC Lyr are too weak to reliably identify. Consequently no differential velocity could be determined.

${ }^{\mathrm{g}}$ Seven light-curve points from unpublished Behlen Observatory photometry are available for V1281 Aql. From these we estimated an epoch of maximum of HJD 2,452,402. The period from the GCVS, 32.05 days, was adopted. 


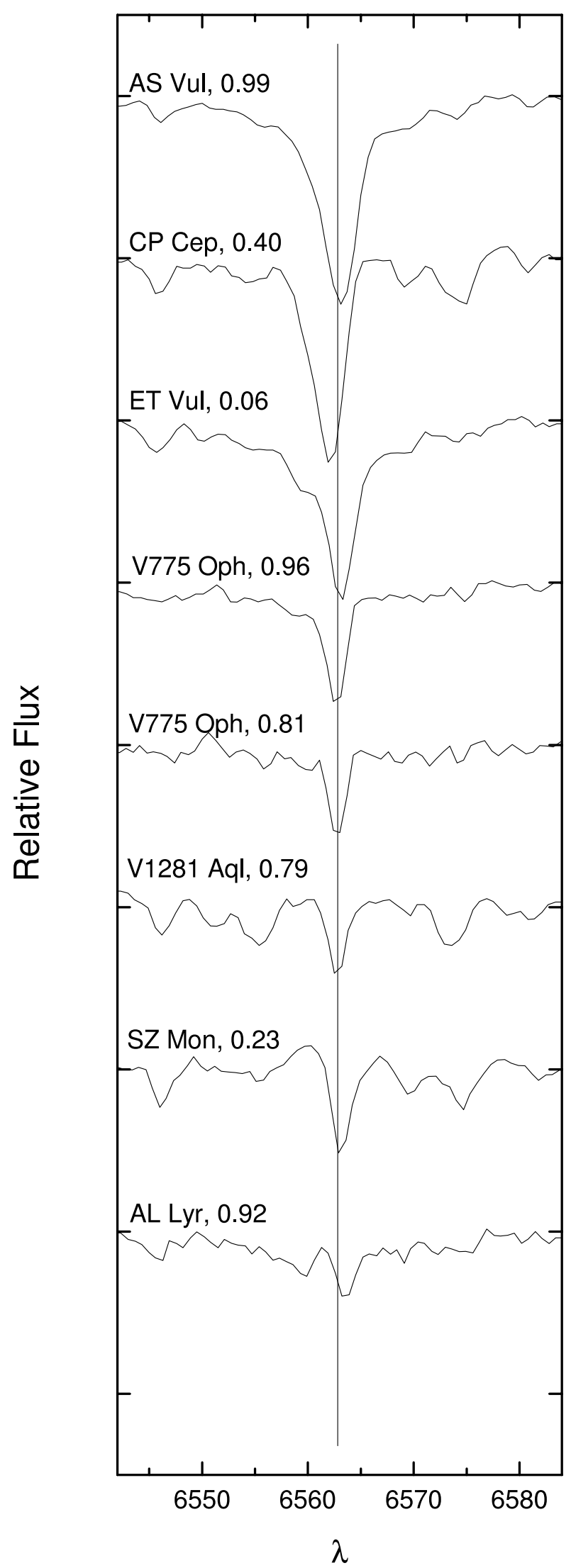

Fig. 1.-Examples of $\mathrm{H} \alpha$ profiles dominated by absorption. Each plot is normalized to a continuum level of 1 and offset vertically from its neighbors for visibility. The tick marks on the ordinate axis are at intervals of 0.5 . Each spectrum is identified by the name of the star and the phase. The vertical line indicates the rest wavelength of $\mathrm{H} \alpha$, and each spectrum has been shifted to the rest frame of the photosphere as defined by the metal lines.

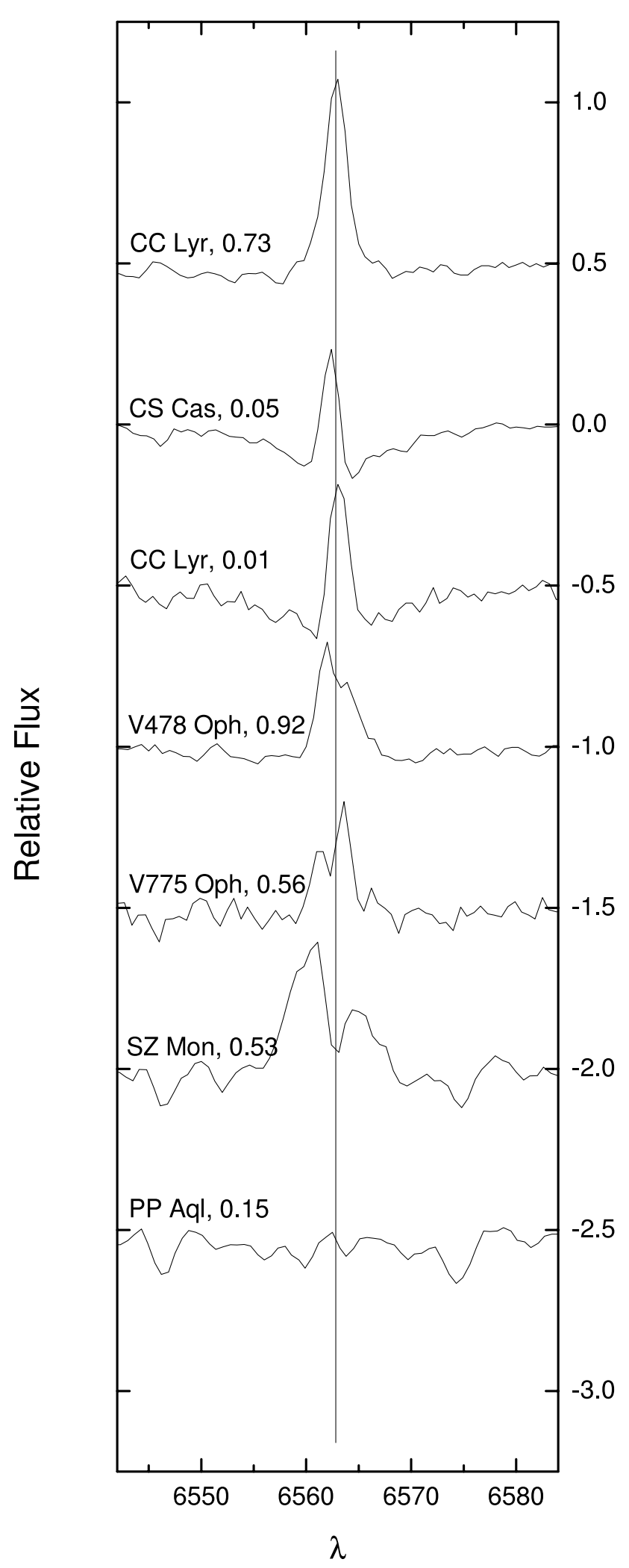

FIG. 2.-Examples of $\mathrm{H} \alpha$ profiles with apparent emission features. The plot has the same format as Fig. 1. 
TABLE 3

Stars with Helium Emission

\begin{tabular}{|c|c|c|c|c|c|}
\hline $\begin{array}{l}\text { Star } \\
(1)\end{array}$ & $\begin{array}{l}\text { Period } \\
\text { (2) }\end{array}$ & $\phi$ of Maximum $\mathrm{H} \alpha$ & $\begin{array}{c}\phi \\
(4)\end{array}$ & $\begin{array}{c}W_{\lambda} \operatorname{He} \lambda 5876 \\
(\AA) \\
(5)\end{array}$ & $\begin{array}{c}\sigma \\
(\AA) \\
(6)\end{array}$ \\
\hline \multirow[t]{2}{*}{ V439 Oph .......... } & 1.9 & 0.81 & 0.84 & -0.10 & 0.02 \\
\hline & $\ldots$ & $\ldots$ & 0.88 & -0.07 & 0.03 \\
\hline \multirow[t]{2}{*}{ AL Lyr ................ } & 13.0 & 0.60 & 0.76 & -0.07 & 0.04 \\
\hline & $\ldots$ & $\ldots$ & 0.84 & -0.13 & 0.05 \\
\hline \multirow[t]{3}{*}{ CS Cas ................. } & 14.7 & 0.70 & 0.77 & -0.12 & 0.03 \\
\hline & $\ldots$ & $\ldots$ & 0.84 & -0.45 & 0.05 \\
\hline & $\ldots$ & $\ldots$ & 0.77 & -0.18 & 0.05 \\
\hline \multirow[t]{4}{*}{ V478 Oph .......... } & 16.3 & 0.68 & 0.68 & -0.16 & 0.03 \\
\hline & $\ldots$ & $\ldots$ & 0.73 & -0.24 & 0.02 \\
\hline & $\ldots$ & $\ldots$ & 0.85 & -0.22 & 0.02 \\
\hline & $\ldots$ & $\ldots$ & 0.92 & -0.16 & 0.03 \\
\hline \multirow[t]{5}{*}{ W Vir .................... } & 17.3 & 0.73 & 0.83 & -0.52 & \\
\hline & $\ldots$ & $\ldots$ & 0.89 & -0.38 & \\
\hline & $\ldots$ & $\ldots$ & 0.95 & -0.38 & \\
\hline & $\ldots$ & $\ldots$ & 0.97 & -0.53 & \\
\hline & $\ldots$ & $\ldots$ & 0.01 & -0.22 & \\
\hline MZ Cyg............... & 21.3 & 0.86 & 0.86 & -0.05 & 0.03 \\
\hline PP Aql ................ & 24.1 & 0.68 & 0.02 & -0.10 & 0.03 \\
\hline \multirow[t]{2}{*}{ TW Cap.............. } & 28.6 & 0.89 & 0.00 & -0.18 & 0.02 \\
\hline & $\ldots$ & $\ldots$ & 0.03 & -0.12 & 0.02 \\
\hline \multirow[t]{3}{*}{ IU Cyg ............... } & 31.4 & 0.95 & 0.98 & -0.12 & 0.02 \\
\hline & $\ldots$ & $\ldots$ & 0.02 & -0.13 & 0.02 \\
\hline & $\ldots$ & $\ldots$ & 0.08 & -0.10 & 0.02 \\
\hline
\end{tabular}

peak strength, is listed in column (7). This quantity is defined as $1-r_{\lambda}$, where $r_{\lambda}$ is the residual intensity at the deepest part of an absorption line or the peak intensity of an emission feature, both in units of the continuum flux. Hence, the line depth is negative for emission. For profiles that are classed as "dE," three velocities and depths are listed. These refer to the shortward emission peak, the minimum between the peaks, and the longward emission peak, respectively.

The equivalent width of $\mathrm{H} \alpha$ is listed in column (8) and is also negative for an emission feature. For profiles with multiple components, the net equivalent width of all of the components is given.

\subsection{Helium Emission}

In spite of our relatively low spectral resolution we should be able to detect He 25876 emission comparable in strength to that found by Raga et al. (1989) and Lèbre \& Gillet (1992) in W Vir. On the other hand, while He $\lambda 6678$ emission has also been reported in W Vir, it is obscured by nearby absorption lines at the resolution of our spectra. Accordingly, we have searched all of our spectra, including those for the shorter period stars discussed in Papers I and II, for emission in He $\lambda 5876$. In each case in which emission appears to be present, the original frames were examined to eliminate spurious detections caused by a cosmic-ray hit or other artifact. In Table 3 we list spectra in which He $\lambda 5876$ emission was detected. We have also added $\mathrm{W}$ Vir using equivalent widths measured from the spectral plots given by Lèbre \& Gillet (1992). Column (1) identifies the star, while the period is listed in column (2). In the third column we give the phase of the spectrum in Table 2 with the strongest $\mathrm{H} \alpha$ emission for each star. In column (4) we identify individual spectra by phase. The equivalent widths of He $\lambda 5876$ are listed in column (5), and estimated uncertainties based on the $\mathrm{S} / \mathrm{N}$ are given in column (6).
Although some of the detections appear marginal, all except one of the spectra listed in Table 3 (V439 Oph at phase 0.88) also exhibit significant $\mathrm{H} \alpha$ emission. Only $25 \%$ of the spectra studied here, or $13 \%$ of all of our spectra, including those discussed in Papers I and II, exhibit $\mathrm{H} \alpha$ emission. This coincidence of helium emission with hydrogen emission strengthens our confidence that we have successfully screened out spurious detections and that those listed are genuine.

\section{DISCUSSION}

\subsection{Differential Velocities}

The difference between the radial velocity of $\mathrm{H} \alpha$ and that of the metal lines, $\Delta \mathrm{Vel}$, is a measure of differential motion within the atmosphere of a pulsating star. For short-period stars the maximum absolute value over the cycle, $|\Delta \mathrm{Vel}|_{\max }$, is useful in distinguishing type II from type I Cepheids (Vinkó et al. 1998; Paper I). To explore this issue among the long-period stars, we have plotted $|\Delta \mathrm{Vel}|_{\max }$ against period in Figure 3. In addition to the values for the present sample of stars from Table 2, we included data from Paper II and from Vinkó et al. (1998). In those cases in which $\mathrm{H} \alpha$ exhibited a double emission line, labeled "dE" in column (4) of Table 2, we have plotted a weighted mean of the velocities of the two emission peaks. It is apparent that $|\Delta \mathrm{Vel}|_{\max }$ is insensitive to whether $\mathrm{H} \alpha$ is in absorption (circles) or emission (triangles).

In Paper II we found that stars with periods between about 4 and 7 days exhibited values of $|\Delta \mathrm{Vel}|_{\max }$ that were relatively small, less than about $25 \mathrm{~km} \mathrm{~s}^{-1}$. However, Figure 19 of Vinkó et al. showed a significant increase at longer periods. Figure 3, with a larger sample of stars, confirms this. However, it is clear that there is no separation between high- $z$ stars and low- $z$ stars. We conclude that differential velocities are not useful for distinguishing type I and type II Cepheids for periods longer than 8 days. 


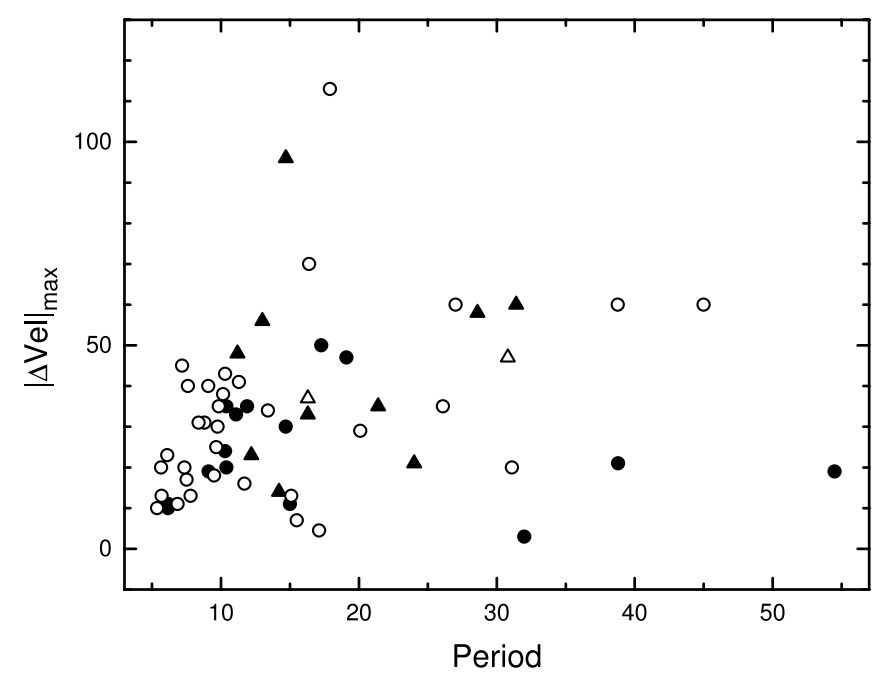

FIG. 3.-Maximum of the absolute value of the $\mathrm{H} \alpha$ differential velocity plotted against the period. The symbols indicate whether $\mathrm{H} \alpha$ is in absorption (circles) or emission (triangles). Open symbols represent low-z stars, and filled symbols represent high- $z$ stars.

\section{2. $\mathrm{H} \alpha$ Emission}

\subsubsection{Emission as a Discriminant of Type}

In Figure 4 we have plotted the equivalent width of $\mathrm{H} \alpha, \mathrm{W}_{\lambda}$, and its line depth against phase. The ordinate is plotted with negative values upward so that emission lines are toward the
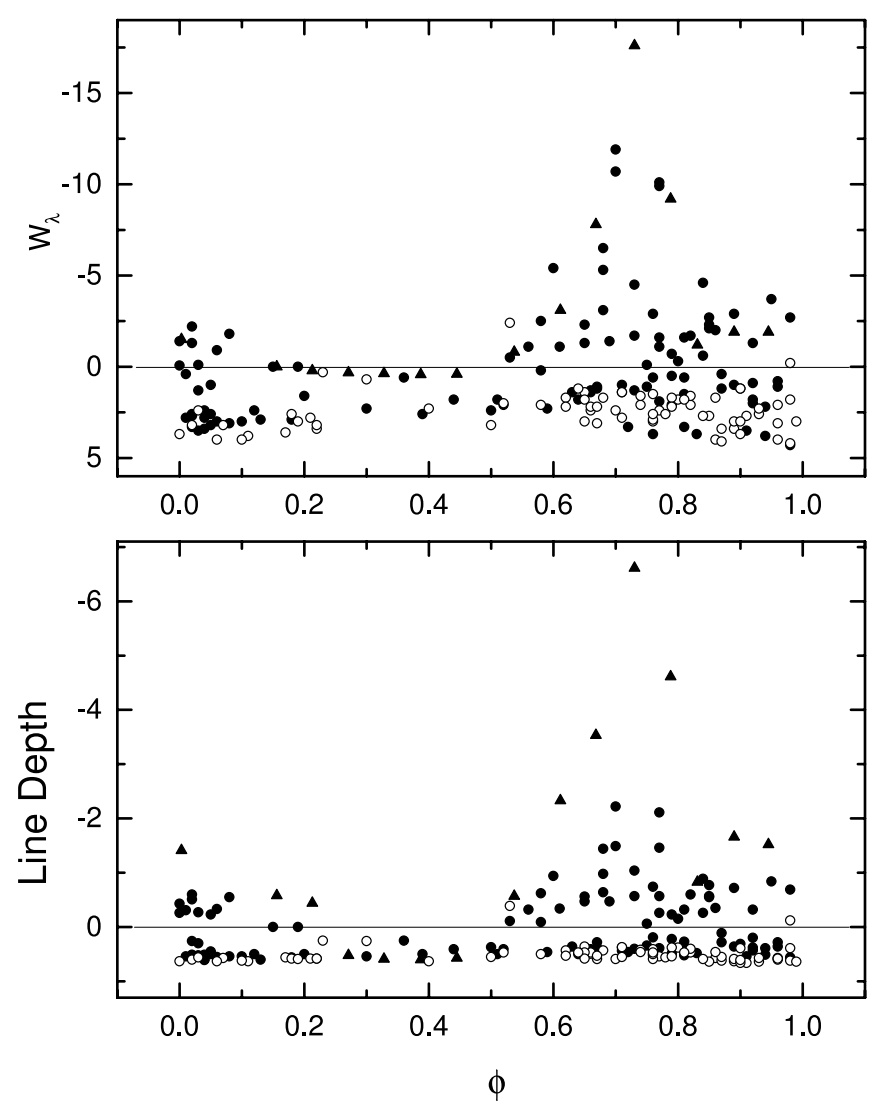

FIG. 4. - Strength of $\mathrm{H} \alpha$ as indicated by the equivalent width (top) and the line depth (bottom) plotted against the phase in the cycle. Open symbols represent low- $z$ stars, and filled symbols represent high- $z$ stars. Values for W Vir are represented by triangles. In both plots, points above the horizontal line indicate emission, while points below correspond to net absorption.
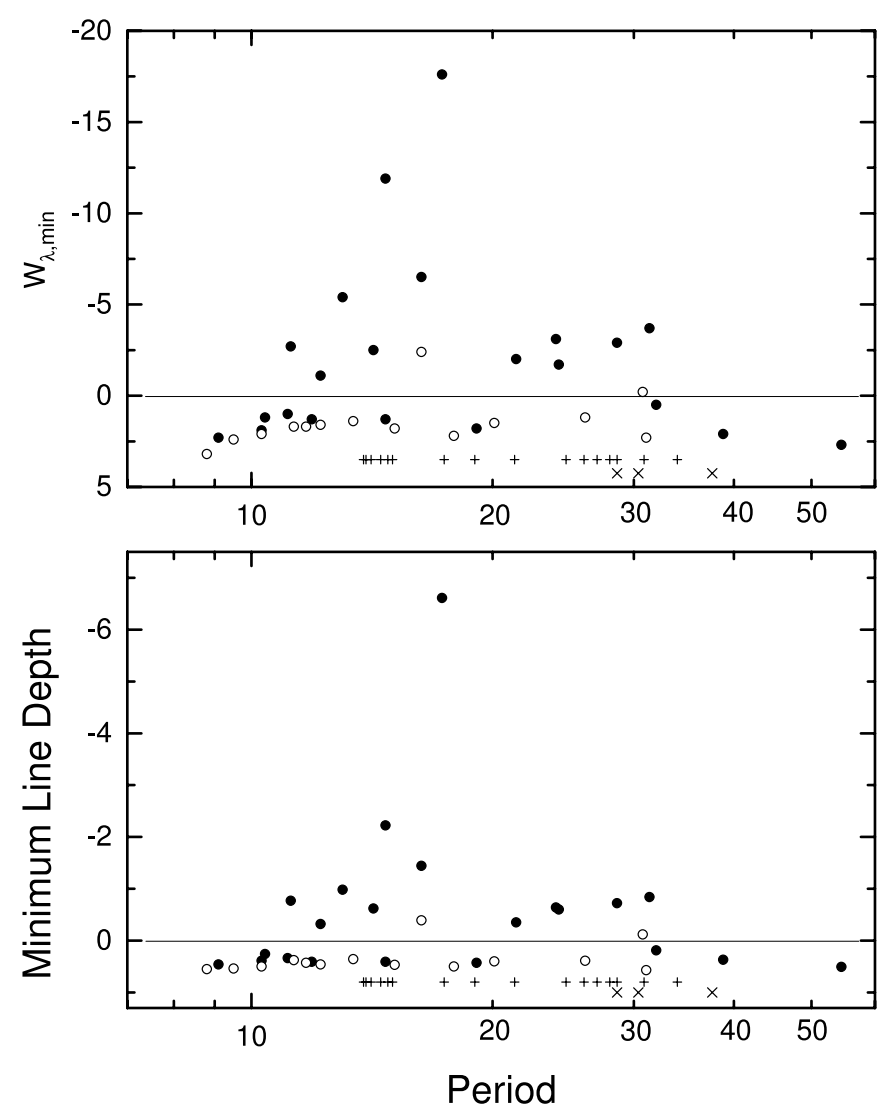

FIG. 5.-Minimum of the equivalent width and the line depth over the cycle plotted against the period. Open symbols denote low- $z$ stars, and filled symbols correspond to high- $z$ stars.

top of the plot. We have also included points (triangles) for W Vir that were measured from Figure 3 of Lèbre \& Gillet (1992). Open symbols represent low- $z$ stars, while filled symbols represent high- $z$ stars.

An inspection of Figure 4 shows that the equivalent widths and line depths for the low- $z$ stars are clustered well below the horizontal line that divides emission from absorption. They have equivalent widths ranging from about $1.2 \AA$ to about $4.3 \AA$ with an obvious phase dependence. The line depths of the low-z stars range from 0.37 to 0.64 and also show some phase dependence. We should expect some type II stars near the Galactic plane, so the open symbols that fall above the bulk of the low $z$ objects are not surprising. The stars in question, SZ Mon, V845 Her, and NN Vul, are classed RVA, CWA, and CWA, respectively, in the GCVS.

The majority of the high- $z$ stars in Figure 4 fall above or only slightly below the horizontal line. While the former are all clearly emission-line objects, the latter are likely to have absorption that is weakened by an emission component. It is interesting to note that the star with the strongest and most persistent emission is W Vir. Even when there is no net emission, in phases from about 0.20 to 0.45 , the profiles of Lèbre \& Gillet clearly show an emission component. Thus, the prototype of the long-period type II Cepheids turns out to be extreme in this regard. Strong emission is very common during the latter part of the cycle in the high- $z$ stars and is detected from about the middle of the cycle until about 0.1 cycles after maximum.

There are a significant number of high- $z$ stars that exhibit absorption lines similar in strength to the low- $z$ stars. To investigate this further, in Figure 5 we present plots of the 
equivalent width and depth of $\mathrm{H} \alpha$ for the spectrum of each star with the strongest emission, or the weakest absorption if no emission was observed. We have omitted EU Cyg and V845 Her because, based on Figure 4, we have no spectra at a time when we would expect emission. Wallerstein (1958) and Harris $\&$ Wallerstein (1984) reported emission or lack of emission in a number of globular cluster and field type II Cepheids. All of the latter are high- $z$ stars. Although they did not give quantitative measures of the strength of the emission, their results will provide further guidance in interpreting our results. Thus, we have plotted plus signs along the bottom of Figure 5 at the periods of stars for which these authors reported emission and crosses at the periods of stars for which they failed to find emission, in spite of having observations at appropriate times.

We note that no star with a period less than 11 days or longer than 34 days exhibits emission. Between 11 and 12 days two of our high- $z$ stars show emission and two do not. Similarly, there is an apparent transition region between 28.6 and 34 days, as revealed by the combined sample. In the region from 12 to 28 days, 21 high- $z$ stars out of 23 from the combined sample have been observed to exhibit emission. Conversely, only one of six low-z stars shows emission. We conclude that in this period range $\mathrm{H} \alpha$ emission is a very reliable discriminator between type I and type II Cepheids.

\subsubsection{High-z Stars with Type I Characteristics}

Schmidt et al. (2004) identified two high- $z$ stars, EU Cyg and DR Cep, with photometric characteristics clearly indicative of type I Cepheids. We have no spectra of EU Cyg in the appropriate part of the cycle for emission, so the present data do not shed further light on the status of that star. However, DR Cep is one of the two high- $z$ stars in Figure 5 with a period between 12 and 28 days that lacks $\mathrm{H} \alpha$ emission. This further strengthens the contention that it is a high- $z$ type I Cepheid.

The other high- $z$ star in the 12 to 28 day range without emission is V833 Oph. Unfortunately, Schmidt et al. (2004) did not obtain photometry for this star and thus did not discuss its status. An examination of the light curve from Berdnikov (1993) generally indicates that V833 Oph is a type II Cepheid; the appearance of the light curve, the light-curve scatter, and the phase parameters, $\phi_{21}$ and $\phi_{31}$, are all consistent with a type II Cepheid, while the amplitude parameters, $R_{31}$ and $R_{41}$, marginally indicate that it is a type I Cepheid. Thus, we conclude that this star is likely to be a type II Cepheid, consistent with its high- $z$ status, and that we simply missed the phases of hydrogen emission.
Schmidt et al. also noted that several other high-z stars, IX Cas, AL Vir, AP Her, and CC Lyr, were possibly type I Cepheids, but they did so with less certainty than was the case for EU Cyg. The first three of these have periods that are less than 11 days, so the spectra cannot confirm this tentative finding. CC Lyr exhibits emission, and we conclude that it is a type II Cepheid.

\subsection{The Prevalence of Helium Emission}

Turning now to the helium emission, we note that the stars in Table 3 cover most of the period range of the W Vir stars and even include one star of much shorter period. Although we do not have enough spectra to define the interval over which there is detectable helium emission, it is clearly present for a smaller part of the cycle than is hydrogen emission.

In AL Lyr we did not detect helium emission in our spectrum at phase 0.80 , even though it was present on spectra earlier and later in the cycle. In W Vir the emission is strongest at phases 0.83 and 0.97 and weaker in between at 0.89 and 0.95 . Since the various spectra are from different cycles, we cannot tell whether the emission goes up and down more than once in a given cycle or changes from one cycle to another. However, taken together these two stars suggest that the situation is more complex than a single shock crossing the atmosphere.

Comparing the phases of strongest hydrogen emission (col. [3] of Table 3) with the phases of helium emission (col. [4]), it is clear that the helium emission occurs later in the cycle than the hydrogen emission. For that reason a simple comparison of the strengths of the two lines at a particular time is unlikely to be meaningful. Raga et al. (1989) and Lèbre \& Gillet (1992) recognized that the behavior of the hydrogen emission is strongly affected by overlying absorption, which further complicates the interpretation of the relative strengths of hydrogen and helium emission. Thus, a full understanding of the hydrogen and helium emission must await the calculation of models that include a detailed treatment of shocks and of the overlying layers of the atmosphere. It will also require higher resolution spectra to obtain more accurate line strengths and profile information. In the meantime, Table 3 provides a list of objects that provide a basis for such an investigation.

We are grateful to the staffs of Kitt Peak National Observatory and Apache Point Observatory for their help in obtaining the data used here. This work is supported in part by NSF grant AST 00-97353.
Berdnikov, L. N. 1993, Perem. Zvezdy, 23, 64

Harris, H. C. 1981a, AJ, 86, 707 $1981 \mathrm{~b}, \mathrm{AJ}, 86,719$ 1985, AJ, 90, 756

Harris, H. C., \& Wallerstein, G. 1984, AJ, 89, 379

Joy, A. H. 1949, ApJ, 110, 105

Kholopov, P. N. 1985, General Catalogue of Variable Stars, Vols. 1 and 2 (4th ed.; Moscow: Nauka) Nauka)

Lèbre, A., \& Gillet, D. 1992, A\&A, 255, 221

\section{REFERENCES}

Meakes, M., Wallerstein, G., \& Opalko, J. F. 1991, AJ, 101, 1795

Raga, A., Wallerstein, G., \& Oke, J. B. 1989, ApJ, 347, 1107

Schmidt, E. G., Johnston, D., Langan, S., \& Lee, K. M. 2004, AJ, 128, 1748

Schmidt, E. G., Langen, S., Lee, K. M., Johnston, D., Newman, P. R., \& Snedden, S. A. 2003a, AJ, 126, 2495 (Paper II)

Schmidt, E. G., Lee, K. M., Johnston, D., Newman, P. R., \& Snedden, S. A. 2003b, AJ, 126, 906 (Paper I)

Schmidt, E. G., \& Seth, A. 1996, AJ, 112, 2769

Vinkó, J., Evans, N. R., Kiss, L. L., \& Szabados, L. 1998, MNRAS, 296, 824 Wallerstein, G. 1958, ApJ, 127, 583

- 1959, ApJ, 130, 560 